\title{
Polysomnographic and Subjective Sleep Predictors of Alcoholic Relapse
}

\author{
Kirk J. Brower, Michael S. Aldrich, and Janette M. Hall
}

\begin{abstract}
Previous studies indicate that subjectively reported and objectively measured sleep abnormalities at baseline can increase the risk of relapse in treated alcoholics. However, previous studies did not include both subjective and objective sleep measures in the same group of patients. We utilized polysomnography and the Sleep Disorders Questionnaire to determine if baseline polysomnography increased the ability to predict relapse beyond the prediction with subjective measures alone, after controlling for nonsleep variables that were associated with relapse. We followed 74 patients with a DSMIII-R diagnosis of alcohol dependence, of whom 36 relapsed to at least some drinking during an average follow-up interval of 5 months. Univariate analyses revealed that relapsed patients did not differ from abstinent patients at baseline in demographics or psychiatric co-morbidity, but they had more prior treatment episodes for alcoholism, more difficulty falling asleep, more complaints of abnormal sleep, and, on polysomnography, longer sleep latencies, shorter rapid eye movement sleep latencies, and less stage 4 sleep percentage than abstinent patients. With a series of logistic regression analyses, which controlled for age and gender, we demonstrated that sleep measures improved the prediction model compared with nonsleep variables alone, and that polysomnography-measured sleep latency was the most significant predictor variable. We conclude that subjective and objective measures of baseline sleep are predictors of relapse in treated alcoholic patients. These data also suggest that neurophysiological dysfunction contributes strongly to the etiology of relapse. Finally, sleep disturbance warrants clinical attention as a target of alcoholism treatment.
\end{abstract}

Key Words: Alcoholism, Alcohol Dependence, Polysomnography, Sleep.

TNESTIGATORS HAVE speculated for $>25$ years that 1 sleep disturbances can predispose to relapse among alcoholic patients. ${ }^{1-3}$ Allen and colleagues, ${ }^{1,4}$ for example, argued that alcoholic patients should have medically supervised hospital care for 7 to 14 days after withdrawal, because disturbances in rapid eye movement (REM) sleep and memory functioning could lead to relapse during that time. However, they did not study drinking outcomes after hospitalization to support their recommendation. Gross

From the Department of Psychiatry and Alcohol Research Center (KJ.B.), and the Department of Neurology, Sleep Disorders Center, and Alcohol Research Center (M.S.A., J.M.H.), University of Michigan, Ann Arbor, Michigan.

Received for publication April 1, 1998; accepted August 3, 1998

This work was supported by Center Grant 5 P50 AA07378 from the National Institute on Alcohol Abuse and Alcoholism. This study was presented in part at the annual meetings of the Research Society on Alcoholism in Washington, D.C., in June 1996 and in San Francisco, CA, in July 1997.

Reprint requests: Kirk J. Brower, M.D., Alcohol Research Center, University of Michigan, 400 East Eisenhower Parkway, Building 2, Suite A, Ann Arbor, MI 48108.

Copyright $(\mathcal{C} 1998$ by The Research Society on Alcoholism. and Hastey ${ }^{2}$ postulated the existence of a "subacute alcohol withdrawal syndrome" characterized by sleep disturbances and craving that could persist for months to years and increase the risk of relapse.

Allen and Wagman ${ }^{5}$ reported that low REM sleep percentage (REM\%) was associated with an increased disposition to drink in a laboratory setting using a behavioral reinforcement paradigm, although experimentally induced REM deprivation failed to increase disposition to drinking. In a subsequent study, Allen and colleagues ${ }^{6}$ reported that low levels of slow wave sleep (SWS) at baseline predicted poor "sobriety" outcome at a 2-month follow-up assessment after discharge from an alcoholism sleep research ward.

Recently, Gillin and colleagues ${ }^{7}$ reported that baseline sleep abnormalities in male alcoholics predicted relapse to drinking within 3 months after discharge from inpatient treatment. Specifically, relapsed patients had increased "REM pressure" (a composite measure including shortened REM sleep latency, increased REM\%, and increased REM density) during hospitalization at baseline when compared with abstainers. There was a nonsignificant trend for decreased SWS\% in the relapsers, compared with abstainers. Interestingly, abstainers and relapsers did not differ in demographic and clinical measures that are usually prognostic, such as severity of dependency, depression, and social stability.

Other researchers have suggested that alcoholics may relapse to drinking in attempts to self-medicate their insomnia. ${ }^{3}$ Studies indicate that about one-third of alcoholics applying to treatment complain of severe insomnia. ${ }^{2} \mathrm{We}$ are aware of only one study, however, that investigated the relationship between subjectively reported sleep disturbance and subsequent drinking in alcoholics. Skoloda et al. ${ }^{8}$ hospitalized patients for 8 weeks, during which time patients rated their sleep each morning. After 2 weeks of a no-drinking period, patients were allowed to drink specified amounts of alcohol while being monitored in the hospital over the next 4 weeks. Patients who drank during the study had reported more sleep disturbance during the first 2 weeks than patients who abstained during the study.

We are unaware of any studies that measured both subjective and objective sleep disturbances as predictors of relapse following treatment for alcoholism. It is unknown whether polysomnography (PSG) increases the ability to predict relapse beyond the prediction with subjective mea- 
sures alone. In the present study, we examined both subjective and objective measures of sleep as predictors of treatment outcome, after controlling for nonsleep variables that can be associated with drinking outcomes in treated alcoholics, such as demographics, severity, and comorbidity. ${ }^{9}$

We hypothesized that (1) nonsleep variables, such as lower education, unmarried status, unemployment, illness severity, and psychiatric co-morbidity, would be associated with relapse; (2) baseline subjective complaints of poor sleep would be associated with relapse; (3) baseline objective measures of sleep would be associated with relapse; and (4) both subjective and objective sleep measures would significantly predict relapse after controlling for nonsleep variables that were associated with relapse.

\section{METHODS}

\section{Subjects}

One hundred and seventy-eight subjects (152 males, 26 females) with DSM-III-R diagnoses of alcohol dependence ${ }^{10}$ were recruited from several closely affiliated alcohol treatment programs that broadly divided into two treatment systems. The first treatment system recruited 86 subjects and consisted of four programs owned and operated by the University of Michigan Medical Center and Chelsea Community Hospital, which formed a joint venture in 1990 to provide addictions treatment. The four programs included an inpatient treatment unit at the University of Michigan Medical Center, a residential treatment program at Chelsea Community Hospital, an older adult inpatient unit at Chelsea Community Hospital, and an outpatient program of the joint venture. These programs are grouped together because of similarities in subject demographics, as well as shared physician and administrative staff. The second treatment system recruited 89 patients and consisted of the inpatient alcohol treatment program at the Ann Arbor Veterans Affairs Hospital (VA system). For three subjects, information on treatment site was missing. Subjects from the University of Michigan Medical Center and Chelsea Community Hospital were more likely than subjects from the VA system to be women ( $29 \%$ vs. $5 \% ; \chi^{2}=7.80, d f=1, p=0.005$ ), to be employed (79\% vs. $27 \%$; $\left.\chi^{2}=16.39, d f=1, p=0.001\right)$, to be married (48\% vs. $8 \% ; \chi^{2}=13.91$, $d f=1, p=0.001)$, to be Caucasian $\left(91 \%\right.$ vs. $74 \% ; \chi^{2}=3.51, d f=1$, $p=0.06)$, and to be younger $(39.3 \pm 11.8$ vs. $42.4 \pm 10.0 \mathrm{yr} ; t=-1.90$, $d f=173, p=0.06)$. Thus, our selection of treatment systems ensured a demographically heterogeneous sample.

Each subject was carefully screened for medical and psychiatric conditions that might influence sleep measurements. Psychiatric diagnoses were determined using a revised version of the Diagnostic Interview Schedule (DIS-IIIR) ${ }^{11}$ which corresponded to DSM-III-R criteria. ${ }^{10}$ Potential subjects were excluded if they had a history of significant medical or psychiatric illness, including psychosis, current major depression, bipolar disorder, borderline personality disorder, heart disease, severe liver disease, seizure disorder (except seizures related to alcohol withdrawal), degenerative or demyelinating central nervous system disease, stroke, transient ischemic attacks, or recent loss of consciousness due to head trauma. A past history of major depression, current dysthymic disorder, or anxiety disorders were not cause for exclusion. Likewise, patients with antisocial personality disorder were not excluded unless severe (i.e., character traits judged by the investigators to interfere with study procedures). Patients were also excluded if they took medications known to affect sleep, including antidepressants, anticonvulsants, antihistamines, neuroleptics, sedative-hypnotics, stimulants, centrally acting antihypertensives ( $\alpha$-methyldopa, reserpine, and clonidine), oral corticosteroids, and theophylline. Patients who met DSM-III-R criteria for abuse or dependence on substances other than alcohol were not excluded, as long as they were substance-free for 2 weeks before their sleep study. Subjects were excluded if they worked night shifts or intentionally stayed awake during usual bedtime hours. All subjects participated in this study only after signing informed consent. The protocol for this study was approved both by the University of Michigan Medical Center Institutional Review Board and the Human Subjects Committee at the VA system.

\section{Procedures}

Patients were evaluated at two points in time: at baseline and at follow-up. The baseline evaluation consisted of a complete medical history, physical examination, a psychiatric diagnostic interview, a structured interview to determine the substance and psychosocial history, and a sleep evaluation. At follow-up, subjects completed a structured interview with a research assistant by either telephone or in person, as determined by the subjects' preference and feasibility. The follow-up interview was conducted between 3 and 12 months after the baseline sleep study. Seventyfour ( $42 \%$ ) of the original 178 subjects completed standardized follow-up interviews an average of 5 months after baseline (mean: 142.7 days, SD: 55.1). Interviews were conducted by trained research assistants of the University of Michigan Alcohol Research Center who were blind to results of the subjects' sleep studies. The follow-up interview assessed the subject's use of alcohol and other drugs, severity of depressive symptoms, and attendance at Alcoholics Anonymous (AA) meetings.

At baseline, subjects underwent one or more nights of nocturnal PSG in the sleep laboratory at the University of Michigan a minimum of 2 weeks after admission to a treatment center. In some cases, subjects were studied from their hospital rooms, which were connected by telemetry to the University of Michigan Sleep Laboratory. The mean time period between PSG and the patient's last drink was 31.5 (SD: 15.5) days. Only data from the first night of sleep monitoring were used for this report, because not all subjects completed more than one night of study.

Sleep was monitored using Sleep Analyzing Computers (Oxford/Microtronics) and standard polysomnographic techniques. Recordings included an electroencephalogram (C3/A2), electrooculogram, submental and anterior tibialis electromyogram, electrocardiogram, respiratory effort (through abdominal and chest strain gauges), airflow (through nose and mouth thermistors), and oxygen saturation (through ear or finger oximetry). Data were digitized using a bedside portable computer and then transmitted to Sleep Analyzing Computers in the control room. The electroencephalogram channel was digitally filtered to yield a nominal band pass of 0.1 to $30 \mathrm{~Hz}$. Data were displayed at a rate of $10 \mathrm{~mm} / \mathrm{sec}$ as a virtual polygraph page on a high-resolution monitor and stored to a hard disk at 250 samples/sec.

Records were masked to remove identifying information so that scorers would be blind to diagnostic and treatment status, then manually scored using 1-min epochs. ${ }^{12}$ Three categories of sleep variables were scored: sleep continuity, sleep architecture, and REM sleep indices. Sleep continuity included total recording period, total sleep time, sleep efficiency $\%$, sleep latency, ${ }^{*}$ and time spent awake after initial sleep onset. Sleep architecture included percentage of net sleep time spent in each stage (stages 1 to 4 and REM sleep). Delta or SWS was calculated by adding stage 3 and stage 4 sleep. REM sleep indices included REM sleep latency, corrected REM sleep latency, $\uparrow$ and REM density.

\section{Instruments and Measures}

The Sleep Disorders Questionnaire (SDQ) is a 175-item, selfadministered instrument that was given at baseline to measure subjective sleep complaints and quality. ${ }^{13}$ Subjects respond to individual questions

* Scoring for sleep latency was defined in this study as the time from start of recording to onset of stages 2 to 4 or REM sleep with a duration of at least $10 \mathrm{~min}$ and with no more than $2 \mathrm{~min}$ of stage 1 or $1 \mathrm{~min}$ of stage $1+1 \mathrm{~min}$ of wakefulness.

+ Corrected REM sleep latency was defined as the time between sleep onset and the first REM period (at least 3 min of REM sleep within 30 min of each other) minus intermittent wakefulness during that interval. 
about the past 6 months (such as "I have trouble getting to sleep at night") by choosing among five Likert-scaled response categories: Never (0), Rarely (1), Sometimes (2), Usually (3), and Always (4). The SDQ yields four clinical-diagnostic scales: (1) sleep apnea, (2) periodic leg movement disorder, (3) psychiatric sleep disorder, and (4) narcolepsy. In addition to the scaled scores, we selected six individual items a priori for analyses that we judged to reflect commonly observed sleep complaints among alcoholics seen in ordinary clinical practice. The items were, "I often have a poor night's sleep" (item 2), "I have trouble getting to sleep at night" (item 3), "My sleep is restless and disturbed" (item 14), "I feel that my sleep is abnormal" (item 44), "I feel that I have insomnia" (item 45), and "I often use alcohol in order to get to sleep" (item 105). For these six items, we used a binary scoring system: subjects who answered "never," "rarely," or "sometimes" were scored as not having the symptom to a clinically significant degree, whereas "usually" and "always" responses were scored as having the symptom.

Psychiatric and substance use diagnoses were determined according to DSM-III-R criteria $^{10}$ using the revised DIS-III-R. ${ }^{11}$ The DIS-III-R also yielded lifetime symptom counts for alcohol dependence (maximum $=9$ ) and antisocial personality disorder, which we used as measures of diagnostic severity. Finally, the age of onset of alcohol dependence was determined by the DIS-III-R and was defined as the age at which the first symptom of the disorder appeared. The Carroll Depression Scale ${ }^{14}$ was administered to assess self-reported depressive symptoms at baseline and at follow-up. Higher scores indicate greater severity of depression. The Michigan Alcoholism Screening Test was used as an index of lifetime drinking problem severity. ${ }^{15}$ The Mini-Mental State Exam was used to assess cognitive functioning. ${ }^{16}$ The total years of heavy drinking over the course of each subjects' lifetime was assessed using a modified version of the Lifetime Drinking History. ${ }^{17}$ Heavy drinking was defined as 28 or more drinks per week for women and 35 or more drinks per week for men.

Follow-up measures included drinking status and patterns, other drug use, depressive symptoms as determined by readministering the Carroll Depression Scale, and attendance at AA meetings. Other drug use was defined as any illicit or nonmedical use of amphetamines, cocaine, marijuana, sedative-hypnotics, opiates, hallucinogens, phencyclidine, or inhalants in the 2 months before interview. Drinking was determined by selfreport using a timeline follow-back interview. ${ }^{18}$ The timeline follow-back method uses a timeline calendar protocol to examine daily alcohol consumption over the follow-up interval using monthly calendars. The reliability and validity of this method have been demonstrated in several studies. $^{18-20}$

\section{RESULTS}

\section{Effects of Follow-up Status}

We conducted univariate analyses comparing subjects whom we followed $(n=74)$ to subjects lost to follow-up $(n=104)$. Before tests of significance, continuous variables were assessed for normality. Nonnormal variables were transformed to approximate normal distributions before conducting further analyses. Continuous variables were analyzed with $t$ tests. Dichotomous variables were tested with either the $\chi^{2}$ test or the Fisher's exact test if cell sizes were too small. All tests were two-tailed.

Significant differences were found between subjects who were followed and subjects who were lost to follow-up for only two of 42 variables (Table 1). Subjects who were followed had engaged in heavy drinking over a longer period of time and had less stage 2 sleep on baseline PSG than subjects who were not followed. Importantly, neither variable was associated with relapse in the followed group.

\section{Outcomes}

The 74 subjects who completed follow-up interviews were categorized into two groups (abstained vs. relapsed) based on their drinking status during the follow-up interval. A relapse was defined as any drinking of alcohol during the follow-up interval. Conversely, abstinence was defined as the absence of drinking any alcohol (i.e., continuous abstinence) during the follow-up interval. Outcome differences between the abstained and relapsed groups were assessed using $t$ tests, Fisher's exact tests, and $\chi^{2}$ tests for univariate analyses.

Slightly more than one-half or 38 of the 74 subjects who completed the follow-up evaluation abstained from alcohol, whereas the other 36 subjects relapsed. Subjects who relapsed drank an average (mean) of $27 \%$ of days and a mean of 2.7 (SD: 4.9) drinks/drinking day during the follow-up interval. During the follow-up interval, subjects who had relapsed attended fewer AA meetings, were less likely to have used other drugs, and had higher depression scores than subjects who had abstained (Table 2). However, differences in drug use and depression scores were not significant after correcting for the number of tests (Bonferroni correction).

\section{Predictors of Relapse}

We first conducted univariate analyses comparing those who relapsed $(n=36)$ to those who abstained $(n=38)$, both for descriptive purposes and to select variables for the regression analyses. Univariate analyses revealed nominally significant differences $(p<0.05)$ between abstainers and relapsers for treatment site, number of prior alcohol treatment episodes, and several sleep variables (Table 3). Treatment at the VA treatment system was associated with relapse. Subjects who relapsed also had more prior treatment episodes than subjects who abstained did. No other demographic, substance-related, or psychiatric differences were found at baseline. Furthermore, there was no statistically significant difference between the abstainers (139 \pm $53 \mathrm{~d})$ and the relapsers $(146 \pm 57 \mathrm{~d})$ in length of the follow-up interval, so the two outcome groups did not differ in their time risk for relapse. Finally, none of the differences in nonsleep variables were significant after correcting for the number of tests (Table 3 ).

Subjects who relapsed reported more subjective sleep disturbance at baseline than subjects who subsequently abstained, including complaints of difficulty falling asleep and abnormal sleep (Table 3). Relapsed subjects also had higher SDQ psychiatric scale scores than abstinent subjects, although no differences between the two outcome groups were found for other SDQ scale scores (narcolepsy, sleep apnea, and periodic leg movement disorder). The SDQ scale score results indicate that the increased sleep complaints of the relapsed patients at baseline were more likely to be associated with psychiatric distress than with sleep disorders, such as narcolepsy, sleep apnea, and periodic leg 
Table 1. Differences Between Followed and Not Followed Groups

\begin{tabular}{|c|c|c|}
\hline Baseline variables & $\begin{array}{l}\text { Not followed group } \\
\qquad(n=104)^{*}\end{array}$ & $\begin{array}{l}\text { Followed group } \\
\qquad(n=74)^{*}\end{array}$ \\
\hline \multicolumn{3}{|l|}{ Demographics and Treatment Site } \\
\hline Age & $40.2 \pm 10.7$ & $41.6 \pm 11.6$ \\
\hline Gender (\% male) & 87 & 84 \\
\hline Race (\% white) & 85 & 82 \\
\hline Education (years) & $12.8 \pm 2.0$ & $13.2 \pm 1.5$ \\
\hline Married $(\%)$ & 33 & 29 \\
\hline Employed (\%) & 57 & 53 \\
\hline Lives alone (\%) & 22 & 23 \\
\hline Treatment site (\% from VA system) & 48 & 53 \\
\hline \multicolumn{3}{|l|}{ Severity of alcohol dependence } \\
\hline Age of Onset & $20.3 \pm 8.2$ & $20.0 \pm 6.7$ \\
\hline Years of heavy drinkingt & $10.7 \pm 8.1$ & $13.8 \pm 10.0$ \\
\hline MAST Score & $40.5 \pm 11.2$ & $42.8 \pm 9.5$ \\
\hline DSM-III-R symptom count & $7.6 \pm 1.6$ & $7.6 \pm 1.5$ \\
\hline Prior alcohol treatments (no.) & $1.8 \pm 1.1$ & $2.2 \pm 2.3$ \\
\hline Family history-positive (1st degree) & $62 \%$ & $60 \%$ \\
\hline \multicolumn{3}{|l|}{ Psychiatric and drug co-morbidity } \\
\hline Carroll Depression Score & $6.9 \pm 5.4$ & $9.0 \pm 7.8$ \\
\hline DSM-III-R Antisocial Symptom Count & $6.2 \pm 3.7$ & $6.2 \pm 4.2$ \\
\hline Antisocial personality disorder & $29 \%$ & $21 \%$ \\
\hline MMSE Score & $28.3 \pm 2.3$ & $28.2 \pm 3.0$ \\
\hline Lifetime drug use disorder (\%) & 45 & 49 \\
\hline Used drugs in past 12 months (\%) & 28 & 30 \\
\hline Tobacco use (pack-years) & $20.3 \pm 19.4$ & $26.0 \pm 19.5$ \\
\hline \multicolumn{3}{|l|}{ SDQ } \\
\hline Psychiatric scale & $36.2 \pm 22.6$ & $40.4 \pm 25.5$ \\
\hline Frequent poor sleep (item 2) & $26.3 \%$ & $25.7 \%$ \\
\hline Difficulty falling asleep (item 3) & $26.3 \%$ & $35.1 \%$ \\
\hline Restless and disturbed sleep (item 14) & $19.2 \%$ & $17.6 \%$ \\
\hline Abnormal sleep (item 44) & $18.2 \%$ & $17.6 \%$ \\
\hline Insomnia (item 45) & $6.1 \%$ & $6.8 \%$ \\
\hline Alcohol used to sleep (item 105) & $39.8 \%$ & $50.0 \%$ \\
\hline \multicolumn{3}{|l|}{ PSG } \\
\hline Total recording period (min) & $384.8 \pm 37.4$ & $384.8 \pm 30.6$ \\
\hline Total sleep time (min) & $311.6 \pm 52.8$ & $301.4 \pm 51.2$ \\
\hline Sleep efficiency (\%) & $81.1 \pm 11.6$ & $78.4 \pm 11.9$ \\
\hline Sleep latency $(\mathrm{min}) \neq$ & $35.4 \pm 35.5$ & $39.3 \pm 32.8$ \\
\hline Wake time after sleep onset (min)‡ & $27.6 \pm 24.7$ & $38.1 \pm 34.7$ \\
\hline Stage $1(\%)$ & $22.3 \pm 11.8$ & $25.2 \pm 14.6$ \\
\hline Stage $2(\%) \dagger$ & $49.1 \pm 11.0$ & $45.1 \pm 13.1$ \\
\hline Stage $3(\%) \ddagger$ & $4.6 \pm 4.9$ & $4.7 \pm 5.1$ \\
\hline Stage $4(\%) \ddagger$ & $3.6 \pm 5.8$ & $4.3 \pm 7.1$ \\
\hline SWS (\%) & $8.2 \pm 8.6$ & $9.0 \pm 10.0$ \\
\hline REM sleep (\%) & $20.5 \pm 6.8$ & $20.6 \pm 6.9$ \\
\hline REM sleep latency (min) & $75.5 \pm 53.4$ & $64.0 \pm 47.2$ \\
\hline REM sleep latency corrected (min) & $70.5 \pm 50.0$ & $60.1 \pm 43.8$ \\
\hline REM density & $1.2 \pm 0.6$ & $1.3 \pm 0.6$ \\
\hline
\end{tabular}

- Means \pm SD are presented. Some variables have smaller sample sizes because of missing data. VA = Veterans Affairs; MAST = Michigan Alcoholism Screening Test; MMSE = Mini-Mental State Exam; SDQ = Sleep Disorders Questionnaire; PSG = Polysomnography.

$\dagger p<0.05$.

$\ddagger$ Statistical test was performed on transformed values not shown in the table.

movement disorder. Polysomnographic measures that distinguished the two subject groups were prolonged sleep latency, decreased stage $4 \%$, and shortened REM latency in the subjects who relapsed (Table 3 ). There was a nonsignificant trend $(p<0.1)$ for the relapse group to have lower sleep efficiency than the abstinent group. Overall, subjects who subsequently relapsed had greater disturbances of both objective and subjective sleep at baseline than subjects who abstained, although only sleep latency was significant after adjusting for the number of tests.

Logistic regression analyses were performed to determine whether baseline sleep measures were useful for predicting relapse after controlling for other clinical variables.
Three domains of predictor variables were considered. The first domain was comprised of nonsleep clinical variables, including demographics, severity of alcohol dependence, psychiatric co-morbidity, and other drug use. The second domain contained subjective sleep measures from the SDQ, and the third domain consisted of objective sleep measures from baseline PSG.

Two series of logistic regression analyses were performed. The preliminary series were used to select variables for the final series. In the preliminary series, we ran separate backward-elimination logistic regression analyses for each variable domain, entering all variables in the domain that significantly differed $(p<0.05)$ between out- 
Table 2. Outcome Differences Between Subjects Who Abstained and Relapsed

\begin{tabular}{lcc}
\hline \multicolumn{1}{c}{ Outcome variables } & $\begin{array}{c}\text { Abstained group } \\
(n=38)^{\star}\end{array}$ & $\begin{array}{c}\text { Relapsed group } \\
(n=36)^{*}\end{array}$ \\
\hline \% days abstinent $\dagger$ & $100 \pm 0.0$ & $73.4 \pm 30.1$ \\
Average drinks/drinking day $\dagger$ & 0 & $2.7 \pm 4.9$ \\
Maximum drinks/drinking dayt & 0 & $15.4 \pm 13.0$ \\
Used other drugs (\%) $\ddagger$ & 25 & 5 \\
Carroll Depression Score at follow-up $\ddagger$ & $6.0 \pm 7.5$ & $10.9 \pm 10.5$ \\
Maximum AA meetings/week (no.) $\ddagger$ & $4.5 \pm 2.8$ & $2.9 \pm 2.7$ \\
AA meetings in past 30 days (no.) $\dagger$ & $10.7 \pm 7.8$ & $5.7 \pm 6.3$ \\
\hline
\end{tabular}

- Means $\pm S D$ are presented. Some variables have smaller sample sizes because of missing data. $\mathrm{AA}=$ Alcoholics Anonymous.

$\dagger p<0.005$. Significant after applying Bonferroni correction $(\rho<0.007)$.

$\mp p<0.05$. Not significant after applying Bonferroni correction $(\rho<0.007)$.

come groups in the univariate analyses. To control for age, gender, and the length of the follow-up interval, these variables were also included in the preliminary regression analyses. However, we did not include treatment site in the regression analyses, because we were interested in patient characteristics, not treatment characteristics that predicted outcome. Moreover, none of the patient characteristics that were associated with relapse (number of prior treatments, sleep latency, stage 4\%, REM sleep latency, and subjective sleep variables) differed between patients recruited from the two treatment systems (data not shown). REM sleep latency and corrected REM sleep latency were highly correlated (Pearson $r=0.98, p=0.0001$ ); thus, only the corrected REM sleep latency was entered because of its higher statistical significance in the univariate analyses ( $p=0.017$ for corrected REM sleep latency vs. $p=0.022$ for REM sleep latency). Finally, to make the odds ratio for corrected REM sleep latency more interpretable, we divided each patient's value for this variable by 10 so we could calculate the odds of relapse for each 10-min increase in corrected REM sleep latency.

The variables selected by domain from the preliminary regression analyses were: number of prior alcohol treatments (nonsleep domain); SDQ question 3, "I have trouble getting to sleep at night" (subjective sleep domain); and sleep latency and corrected REM sleep latency (objective sleep domain). A double dagger in Table 3 represents the selected variables.

For the final series of logistic regression analyses, we examined four models. The models differed in the number and order of domains entered (Table 4). To examine the effect of adding polysomnography to information gathered in routine clinical interviews, we first entered the nonsleep domain (model 1), then the subjective sleep domain (model $2 \mathrm{a}$ ), and finally the objective sleep domain (model 3 ). This order of entry was chosen to determine if sleep measures added predictive ability after controlling for other clinical variables known to be associated with treatment outcome. Of the sleep measures, subjective sleep variables were entered before objective sleep variables to determine if PSG added predictive ability to information ordinarily gathered as part of routine clinical interviews. Once a variable was entered into the analysis, it was forced to stay in the model.
Table 3. Baseline Differences as a Function of Outcome Group

\begin{tabular}{|c|c|c|}
\hline Baseline predictor variables & $\begin{array}{l}\text { Abstained group } \\
(n=38)^{*}\end{array}$ & $\begin{array}{l}\text { Relapsed group } \\
\qquad(n=36)^{*}\end{array}$ \\
\hline \multicolumn{3}{|l|}{ Demographics and treatment site } \\
\hline Age & $40.7 \pm 11.8$ & $42.5 \pm 11.5$ \\
\hline Gender ( $\%$ male) & 76 & 92 \\
\hline Race (\% white) & 82 & 83 \\
\hline Education (years) & $13.5 \pm 1.6$ & $12.8 \pm 1.4$ \\
\hline Married (\%) & 34 & 23 \\
\hline Employed (\%) & 56 & 50 \\
\hline Lives alone (\%) & 20 & 26 \\
\hline Treatment site (\% from VA system)† & 37 & 71 \\
\hline \multicolumn{3}{|l|}{ Severity of alcohol dependence } \\
\hline Age of onset & $20.2 \pm 8.2$ & $19.8 \pm 4.9$ \\
\hline Years of heavy drinking & $13.2 \pm 10.2$ & $14.3 \pm 10.0$ \\
\hline MAST Score & $43.0 \pm 9.4$ & $42.5 \pm 9.6$ \\
\hline DSM-III-R symptom count & $7.4 \pm 1.4$ & $7.7 \pm 1.5$ \\
\hline Prior alcohol treatments $\ddagger$ (no.) $\S$ & $1.6 \pm 0.8$ & $2.9 \pm 3.0$ \\
\hline Family history-positive (1st degree) & $66 \%$ & $54 \%$ \\
\hline \multicolumn{3}{|l|}{ Psychiatric and drug co-morbidity } \\
\hline Carroll Depression Score & $7.6 \pm 7.6$ & $10.5 \pm 7.8$ \\
\hline Antisocial symptom count & $5.6 \pm 4.2$ & $6.8 \pm 4.2$ \\
\hline Antisocial personality disorder & $15 \%$ & $29 \%$ \\
\hline MMSE Score & $27.8 \pm 3.6$ & $28.5 \pm 2.2$ \\
\hline Lifetime drug use disorder (\%) & 60 & 37 \\
\hline Used drugs in past 12 months (\%) & 39 & 20 \\
\hline Tobacco use (pack-years) & $22.1 \pm 23.0$ & $30.0 \pm 14.2$ \\
\hline \multicolumn{3}{|l|}{ SDQ } \\
\hline Psychiatric Scale§ & $34.6 \pm 26.2$ & $46.5 \pm 23.6$ \\
\hline Frequent poor sleep (item 2) & $21.0 \%$ & $30.6 \%$ \\
\hline Difficulty falling asleep $\ddagger$ (item 3)† & $21.0 \%$ & $50.0 \%$ \\
\hline Restless and disturbed sleep (item 14) & $15.8 \%$ & $19.4 \%$ \\
\hline Abnormal sleep (item 44$) \S$ & $7.9 \%$ & $25.0 \%$ \\
\hline Insomnia (item 45) & $2.6 \%$ & $13.9 \%$ \\
\hline Alcohol used to sleep (item 105) & $39.5 \%$ & $61.1 \%$ \\
\hline \multicolumn{3}{|l|}{ PSG } \\
\hline Total recording period (min) & $383.0 \pm 30.8$ & $386.6 \pm 30.6$ \\
\hline Total sleep time $(\mathrm{min})$ & $309.0 \pm 51.4$ & $293.4 \pm 50.4$ \\
\hline Sleep efficiency (\%) & $80.8 \pm 12.1$ & $75.8 \pm 11.3$ \\
\hline Sleep latency $¥(\min ) \dagger, \#$ & $29.1 \pm 25.0$ & $50.0 \pm 36.7$ \\
\hline Wake time after sleep onset (min)\# & $38.5 \pm 35.9$ & $37.7 \pm 34.0$ \\
\hline Stage $1(\%)$ & $23.8 \pm 13.2$ & $26.7 \pm 15.9$ \\
\hline Stage $2(\%)$ & $46.4 \pm 12.6$ & $43.8 \pm 13.7$ \\
\hline Stage $3(\%) \#$ & $4.5 \pm 4.6$ & $4.9 \pm 5.7$ \\
\hline Stage $4(\%) \S$, \# & $5.6 \pm 8.0$ & $2.9 \pm 5.9$ \\
\hline SWS (\%) & $10.1 \pm 10.3$ & $7.8 \pm 9.8$ \\
\hline REM sleep (\%) & $19.7 \pm 7.4$ & $21.7 \pm 6.1$ \\
\hline REM sleep latency $(\min ) \S$ & $76.0 \pm 53.0$ & $51.3 \pm 36.9$ \\
\hline REM sleep latency corrected $¥(\min ) \S$ & $71.8 \pm 49.8$ & $47.8 \pm 32.8$ \\
\hline REM density & $1.3 \pm 0.6$ & $1.2 \pm 0.5$ \\
\hline
\end{tabular}

Note: After correcting for the number of tests in each domain (nonsleep, subjective sleep, and objective sleep domains), only sleep latency remained statistically significant $(\rho=0.0024)$.

* Means \pm SD are presented. Some variables have smaller sample sizes because of missing data. VA = Veterans Affairs; MAST = Michigan Alcoholism Screening Test; MMSE = Mini-Mental State Exam.

$t p<0.01$.

$¥$ Variables selected for final series of logistic regression models. $\S p<0.05$.

\# Statistical test was performed on transformed values not shown in the table.

After each variable domain was entered, we determined the overall significance of the model, the percentage of cases correctly classified, the amount of variance accounted for, and the odds ratios for each variable (Table 4).

In model 1, the nonsleep variable (number of prior treatments) correctly predicted drinking status at follow-up for $64 \%$ of the sample and accounted for $15 \%$ of the variance as measured by the Nagelkerke ${ }^{21} R^{2}$. The $\chi^{2}$ for the model was $8.6(d f=1, p=0.0034)$. Adding the subjective sleep 
Table 4. Final Logistic Regression Models

\begin{tabular}{|c|c|c|c|c|}
\hline Statistical measure & $\begin{array}{c}\text { Model } \\
1 \dagger\end{array}$ & $\begin{array}{c}\text { Model } \\
2 a \neq\end{array}$ & $\begin{array}{l}\text { Model } \\
2 \mathrm{~b} \S\end{array}$ & $\begin{array}{c}\text { Model } \\
3 \#\end{array}$ \\
\hline \multicolumn{5}{|l|}{ Odds ratios for predictor variables } \\
\hline Prior treatment episodes & $1.56^{*}$ & 1.42 & 1.46 & 1.35 \\
\hline Trouble falling asleep (SDQ item 3) & - & $3.12^{\star}$ & - & 2.88 \\
\hline Corrected REM sleep latency & - & - & 0.89 & 0.88 \\
\hline Sleep latency & - & - & $2.42^{\star \star}$ & $2.35^{\star}$ \\
\hline$\%$ of cases correctly predicted & 64 & 72 & 68 & 72 \\
\hline Model $\chi^{2} p$ value & 0.0034 & 0.0014 & 0.0001 & 0.0001 \\
\hline
\end{tabular}

$p$ values are as follows:

" significance level of $\chi^{2}$ statistic for this variable is $p<0.05$; ** significance level of $\chi^{2}$ statistic for this variable is $p<0.01$.

t Only nonsleep domain entered.

$\ddagger$ Subjective sleep domain added to model 1 to model the effect of interviewgathered variables.

$\S$ Objective sleep domain added to model 1 to model the etiology of relapse. \# All three domains are forced into the model.

variable (SDQ item 3) to model 1 resulted in model $2 a$, which predicted drinking status for $72 \%$ of the sample and accounted for $22 \%$ of the variance. The model $\chi^{2}$ increased to 13.1 ( $d f=2, p=0.0014$ ). When all three domains were forced into the regression, model 3 predicted drinking status for $72 \%$ of the sample and accounted for $37 \%$ of the variance. The model $\chi^{2}$ increased to $23.7(d f=4, p=$ 0.0001 ).

To examine processes involved in the etiology of relapse, we first entered the most distal variable, which was the number of prior treatment episodes (model 1 again). Patients who presented for their current treatment episode with a history of past treatment failures were assumed to have increased illness severity that resulted in treatment resistance. Although illness severity and treatment resistance may have a neurophysiological basis, our only neurophysiological measure, PSG, was a measure of current dysfunction that did not necessarily precede the previous treatment episodes. However, we cannot rule out on the basis of this study that the neurophysiological dysfunction that we measured via PSG did not precede the previous treatment failures. Nevertheless, we entered the objective sleep domain second (model $2 b$ ), because we reasoned that as a current neurophysiological measure, it was more proximal to study outcome than past history. We entered the subjective sleep domain last (model 3 again) because we interpreted subjective sleep as an indirect measure of objective sleep that is influenced by psychological factors. A modest but significant correlation between trouble falling asleep (SDQ item 3$)$ and sleep latency $(r=0.25, p=0.03)$ supported our assumption that the subjective and objective sleep variables, respectively, were measuring a similar process. Thus, we viewed objective sleep as the neurophysiological basis of subjective sleep; consequently, objective sleep was conceptualized as a more primary, etiological predictor of relapse than subjective sleep. When the objective sleep variables were added to model 1 , model $2 b$ resulted, which predicted drinking status for $68 \%$ of the sample and accounted for $32 \%$ of the variance. The model $\chi^{2}$ was $20.5(d f=3, p=0.0001)$.
The odds ratios for variables in each step of the model are shown in Table 4 . The odds ratios changed very little after adding each subsequent domain. The final model (model 3) shows that each additional episode of prior treatment increased the odds of relapse by 1.4 -fold. Having difficulty falling asleep increased the odds of relapse by 2.9-fold. Also, the odds of relapse decreased by a factor of 0.9 for every 10 -min increase in corrected REM sleep latency. Finally, the sleep latency variable was transformed using a natural logarithm; thus, each $1 \%$ increase in sleep latency yielded a $2.4 \%$ increase in the odds of relapse.

\section{DISCUSSION}

We found support for our hypothesis that nonsleep variables would be associated with relapse, although demographics and co-morbidity were not. Only the number of prior alcohol treatments, which we interpreted as a measure of treatment resistance and illness severity, was significantly associated with relapse in our sample. Likewise, we found that both subjective and objective sleep measures predicted relapse after treatment for alcoholism even after controlling for prior alcohol treatments. In short, patients who relapsed had worse sleep at baseline than patients who abstained during a 5-month follow-up period.

Our results are consistent with those of Gillin et al. ${ }^{7}$ in showing an effect of shortened REM sleep latency on alcoholic relapse, although we did not find an effect for REM\% or REM density. In Gillin's study, increased REM density predicted relapse in just 1 of 2 samples tested. Likewise, we reported preliminary findings in which increased REM\% and decreased SWS\% were predictors of relapse ${ }^{22,23}$ but those sample sizes were smaller than in this study and previously we did not control for all the clinical variables that we analyzed in this study. The work of Allen et al., ${ }^{6}$ although also suggestive of a predictive effect of decreased SWS on relapse, involved a small sample size, poorly defined outcome measures, and a lack of control for confounding clinical variables, such as age and severity of dependence. We tentatively, conclude, therefore, that SWS, REM\%, and REM density are inconsistent and unstable predictors of relapse, in contrast to shortened REM sleep latency that was a consistent predictor across two recent studies.

Both objective and subjective measures of difficulty falling asleep at night were associated with relapse to drinking. Subjectively reported trouble falling asleep predicted drinking outcomes in at least one other study. ${ }^{8}$ However, PSG was not performed in that study, so subjects may have been responding to sleep questionnaires with generalized dissatisfaction about their lives or specific misperceptions about their sleep. The present study is the first to demonstrate that both objectively measured and subjectively reported sleep disturbances can predict relapse in the same group of alcoholic patients. Moreover, objective sleep disturbance predicted relapse, even after controlling for sub- 
jective sleep measures and other clinical variables. In particular, PSG-measured sleep latency was the most significant predictor variable.

Patients are often told by clinicians that sleep disturbances are common during early recovery and will improve with abstinence. The assumption is that if treatment focuses on the goal of abstinence, then sleep will improve naturally without further intervention. Unfortunately, this assumption may only be partially correct. For example, some patients manifest persistent sleep abnormalities despite prolonged abstinence. ${ }^{24-26}$ Moreover, accumulating evidence now suggests that alcoholic patients with the most disturbed sleep are most likely to relapse. Therefore, we recommend that sleep disturbances be monitored closely with careful consideration of both behavioral ${ }^{27-29}$ and pharmacological treatments. It is tempting to speculate that antidepressants, most of which are known to prolong REM sleep latency,$^{30}$ may be useful in preventing relapse in alcoholic patients with sleep disturbances. It is also possible that sedating antidepressants, such as trazodone and mirtazapine, might prevent relapse by shortening sleep latency in selected patients. Benzodiazepines and other crossdependent sedative-hypnotics are probably best avoided in alcoholics (except for short-term detoxification) due to their toxicity when combined with alcohol and their potential for abuse by alcoholic patients. ${ }^{31,32}$

Several methodological concerns must be considered when interpreting these results. First, relapse status, the treatment outcome variable of interest, was determined solely by self-report without biochemical or other corroboration, such as by a friend or family member. Second, relapse status was the only outcome variable investigated, and our definition of relapse was very stringent, not allowing for any drinking during the follow-up period. The fact that sleep disturbance can predict a return to drinking may be less clinically valuable than predicting a "full" relapse with hazardous drinking and adverse consequences. Future studies should use multidimensional outcome measures. Third, only one night of sleep was recorded, which did not allow subjects to adjust to the "first night effect" of sleeping under novel conditions. Fourth, patients were followed over differing time periods. However, the length of the follow-up period did not differ between the two outcome groups, and it did not predict relapse in the preliminary regression analyses. Fifth, patients did not receive uniform treatments. Indeed, we found that treatment site was significantly associated with outcome. Nevertheless, other variables that predicted outcome did not differ between treatment sites. Moreover, the use of diverse treatment sites ensured a heterogeneous sample and may increase the generalizability of the results.

Sixth, only $42 \%$ of patients receiving baseline sleep assessments were followed over time and were included in our analyses of relapse predictors. The low follow-up rate reflects the fact that longitudinal outcomes were added as a secondary area of interest after starting our primary investigations on the effects of alcoholism and aging on sleep abnormalities. ${ }^{33}$ Although the representativeness of the followed sample may be questioned, followed and not followed patients did not differ on any baseline variables that predicted outcome. Nevertheless, they did differ on the percentage of stage 2 sleep and the duration of heavy drinking. Moreover, one reasonably assumes that a "lostto-follow-up" group has a greater proportion of relapsed patients than a followed group. On the other hand, we have no theoretical reason or empirical evidence from the existing literature to hypothesize that the percentage of stage 2 sleep is related to alcoholic relapse. Regarding duration of heavy drinking, we interpreted this variable as a measure of illness severity, and we hypothesized that increased illness severity would be related to relapse. Yet, we paradoxically found fewer years of heavy drinking in the not followed group. Thus, the available data suggest that the potential compromise to study integrity because of this problem is low. Nevertheless, our results must be interpreted with caution until replicated by other studies with better follow-up rates, more consistent follow-up intervals, better characterization of treatment variables, and multidimensional outcome measures that are corroborated.

Strengths of this study include a relatively large sample size for a polysomnographic investigation of alcoholic patients, co-measurement of both subjectively reported and objectively measured sleep in the same group of patients, and a heterogeneous sample that included women, patients with commercial insurance, and patients treated in a VA Medical Center.

In summary, both subjective and objective measures of baseline sleep were associated with relapse in treated alcoholic patients. The ability of PSG to predict outcome status after controlling for other variables reflects the etiological importance of neurophysiological factors in the relapse process. Finally, sleep disturbances warrant clinical attention as a target of treatment and should be considered in alcoholism treatment trials.

\section{ADDENDUM}

Since the submission of this article, two other articles were recently published that provide further evidence that sleep abnormalities are predictors of alcoholic relapse. ${ }^{34,35}$

\section{ACKNOWLEDGMENTS}

We thank Drs. Elizabeth Hill and Myra Kim for statistical consultation, and Dr. Robert Zucker for his helpful review of the manuscript.

\section{REFERENCES}

1. Allen RP, Faillace LA, Wagman A: Recovery time for alcoholics after prolonged alcohol intoxication. Johns Hopkins Med J 128:158-164, 1971

2. Gross MM, Hastey JM: Sleep disturbances in alcoholism, in Tarter RE, Sugarman AA (eds): Alcoholism: Interdisciplinary Approaches to an Enduring Problem. Reading, MA, Addison-Wesley, 1976, p 257 
3. Vitiello MV: Sleep, alcohol, and alcohol abuse. Addict Biol 2:151158,1997

4. Allen RP, Wagman A, Faillace LA, MacIntosh M: Electroencephalographic (EEG) sleep recovery following prolonged alcohol intoxication in alcoholics. J Nerv Ment Dis 153:424-433, 1971

5. Allen RP, Wagman AM: Do sleep patterns relate to the desire for alcohol? Adv Exp Med Biol 59:495-508, 1975

6. Allen RP, Wagman AMI, Funderburk FR: Slow wave sleep changes: Alcohol tolerance and treatment implications. Adv Exp Med Biol 85A:629-640, 1977

7. Gillin JC, Smith TL, Irwin M, Butters N, Demodena A, Schuckit M: Increased pressure for rapid eye movement sleep at time of hospital admission predicts relapse in nondepressed patients with primary alcoholism at 3-month follow-up. Arch Gen Psychiatry 51:189-197, 1994

8. Skoloda TE, Alterman AI, Gottheil E: Sleep quality reported by drinking and non-drinking alcoholics, in Gottheil EL (ed): Addiction Research and Treatment: Converging Trends. Elmsford, NY, Pergamon, 1979, p 102

9. Brower KJ, Blow FC, Hill EM, Mudd SA: Treatment outcome of alcoholics with and without cocaine disorders. Alcohol Clin Exp Res 18:734-739, 1994

10. American Psychiatric Association: DSM-III-R: Diagnostic and Statistical Manual of Mental Disorders (ed 3-revised). Washington, D.C., American Psychiatric Association, 1987

11. Robins LN, Helzer JE, Croughan J, Ratcliff KS: National Institute of Mental Health Diagnostic Interview Schedule: Its history, characteristics, and validity. Arch Gen Psychiatry 38:381-389, 1981

12. Rechtschaffen A, Kales AA: A Manual of Standardized Terminology, Techniques and Scoring System for Sleep Stages of Human Subjects. Washington, D.C., Government Printing Office (NIH Publication No. 204), 1968

13. Douglass $A B$, Bornstein $R$, Nino-Murcia G, Keenan S, Miles L, Zarcone V Jr, Guilleminault C, Dement WC: The Sleep Disorders Questionnaire. I. Creation and multivariate structure of SDQ. Sleep 17:160167, 1994

14. Carroll BJ, Feinberg M, Smouse PE, Rawson SG, Greden JF: The Carroll Rating Scale for Depression. I. Development, reliability and validation. Br J Psychiatry 138:194-200, 1981

15. Selzer ML: The Michigan Alcoholism Screening Test (MAST): The quest for a new diagnostic instrument. Am J Psychiatry 127:1653-1658, 1971

16. Folstein MF, Folstein SE, McHugh PR: "Mini-mental state": A practical method for grading the cognitive state of patients for the clinician. J Psychiatr Res 12:189-198, 1975

17. Skinner HA, Sheu W: Reliability of alcohol use indices: The Lifetime Drinking History and the MAST. J Stud Alcohol 43:1157-1170, 1982

18. Sobell LC, Sobell MB, Leo GI, Cancilla A: Reliability of a timeline method: Assessing normal drinkers' reports of recent drinking and a comparative evaluation across several populations. Br J Addict 83:393-
402,1988

19. Maisto SA, Sobell MB, Cooper AM, Sobell LC: Test-retest reliability of retrospective self-reports in three populations of alcohol abusers. J Behav Assess 1:315-326, 1979

20. Sobell LC, Maisto SA, Sobell MB, Cooper AM: Reliability of alcohol abuser's self-reports of drinking behavior. Behav Res Ther 17: $157-160,1979$

21. Nagelkerke NJD: A note on a general definition of the coefficient of determination. Biometrika 78:691-692, 1991

22. Aldrich MS, O'Neal EA, Eiser AS, Kroll P, Brower K, Shipley JE: Slow wave sleep decrement and relapse tendency in alcoholics in treatment. Sleep Res 23:185, 1994 (abstr)

23. Aldrich MS, Hall JM, Eiser AS, Kroll P, Brower K, Shipley JE: Slow wave sleep and REM sleep as predictors of relapse in alcoholics. Alcohol Clin Exp Res 20(Suppl. 2):140A, 1996 (abstr)

24. Adamson J, Burdick AJ: Sleep of dry alcoholics. Arch Gen Psychiatry $28: 146-149,1973$

25. Drummond SPA, Gillin JC, Irwin M, Smith T, Golshon S, Demodena A, Danowski S, Schuckit M: Sleep changes in patients with primary alcoholism after one year of abstinence. Sleep Res 24:159, 1995 (abstr)

26. Williams HL, Rundell OH: Altered sleep physiology in chronic alcoholics: Reversal with abstinence. Alcohol Clin Exp Res 2:318-325, 1981

27. Kupfer DJ, Reynolds CFI: Management of insomnia. N Engl J Med 336:341-346, 1997

28. Stepanski EJ: Behavioral therapy for insomnia, in Kryger $\mathbf{M H}$, Roth T, Dement WC (eds): Principles and Practice of Sleep Medicine, ed 2. Philadelphia, Saunders, 1994, p 535

29. Zarcone VP Jr: Sleep hygiene, in Kryger MH, Roth T, Dement WC (eds): Principles and Practice of Sleep Medicine, ed 2. Philadelphia, Saunders, 1994, p 542

30. Obermeyer WH, Benca RM: Effects of drugs on sleep. Neurol Clin 14:827-840, 1996

31. Kranzler HR: Evaluation and treatment of anxiety symptoms and disorders in alcoholics. J Clin Psychiatry 57:15-21, 1996

32. Ciraulo DA, Sarid-Segal O: Benzodiazepines: Abuse liability, in Cowley DS, Roy-Byrne PP (eds): Benzodiazepines in Clinical Practice: Risks and Benefits. Washington, D.C., American Psychiatric Press, 1991, p 157

33. Aldrich MS, Shipley JE, Tandon R, Kroll PD, Brower KJ: Sleepdisordered breathing in alcoholics: Association with age. Alcohol Clin Exp Res 17:1179-1183, 1993

34. Clark CP, Gillin JC, Golshan S, Demodena A, Smith TL, Danowski S, Irwin M, Schuckit M: Increased REM sleep density at admission predicts relapse by three months in primary alcoholics with a lifetime diagnosis of secondary depression. Biol Psychiatry 43:601607,1998

35. Peters PJ, Foster J: Sleep disturbance in alcohol misuse: A predictor of relapse. Alcohol Clin Exp Res 22(Suppl.):183A, 1998 (abstr) 